\title{
The smiling scan technique: Facially driven guided surgery and prosthetics
}

\author{
4 Q1 Alessandro Pozzi ${ }^{\mathrm{a}, \mathrm{b}, *}$, Lorenzo Arcuri ${ }^{\mathrm{c}}$, Peter K. Moy ${ }^{\mathrm{d}}$ \\ a Dental College of Georgia, Augusta University, USA \\ ${ }^{\mathrm{b}}$ Private Practice, Rome, Italy \\ ${ }^{\mathrm{C}}$ University of Rome Tor Vergata, Italy \\ d Department of Oral \& Maxillofacial Surgery, UCLA School of Dentistry, Los Angeles, CA, USA
}

\section{A R T I C L E I N F O}

\section{Article history:}

Received 21 October 2017

Received in revised form 24 February 2018

Accepted 1 March 2018

Available online xxx

\section{Keywords:}

Digital workflow

Smile design

Computer assisted surgery

Computer guided surgery

CAD/CAM

Dental implant

\begin{abstract}
A B S T R A C T
Purpose: To introduce a proof of concept technique and new integrated workflow to optimize the functional and esthetic outcome of the implant-supported restorations by means of a 3-dimensional (3D) facially-driven, digital assisted treatment plan.

Methods: The Smiling Scan technique permits the creation of a virtual dental patient (VDP) showing a broad smile under static conditions. The patient is exposed to a cone beam computed tomography scan $(\mathrm{CBCT})$, displaying a broad smile for the duration of the examination. Intraoral optical surface scanning (IOS) of the dental and soft tissue anatomy or extraoral optical surface scanning (EOS) of the study casts are achieved. The superimposition of the digital imaging and communications in medicine (DICOM) files with standard tessellation language (STL) files is performed using the virtual planning software program permitting the creation of a VDP.

Conclusions: The smiling scan is an effective, easy to use, and low-cost technique to develop a more comprehensive and simplified facially driven computer-assisted treatment plan, allowing a prosthetically driven implant placement and the delivery of an immediate computer aided design (CAD) computer aided manufacturing (CAM) temporary fixed dental prostheses (CAD/CAM technology).
\end{abstract}

(C) 2018 Published by Elsevier Ltd on behalf of Japan Prosthodontic Society.

\section{Introduction}

The growing interest in minimally invasive implant placement with the option of delivering a pre-fabricated provisional prosthesis immediately, have led to the development of numerous 3-Dimensional (3D) planning software programs [1-6]. The new technological advancements have significantly improved data acquisition, leading to a more realistic and accurate overview of the bony and anatomic structures, as well as bone density, to predict the stability of implants starting with the virtual planning stage [7]. Performing a prosthetically driven diagnosis and treatment are mandatory to achieve optimal implant positioning and delivering the ideal prosthetic contour $[8,9]$. The $3 \mathrm{D}$ visualization of the implant recipient site characteristics and neighboring anatomy provides the clinician with more insight into the surgical, prosthetic and aesthetic requirements of the treatment and may improve decision-making, increasing the predictability of the overall implant treatment [10]. The facial analysis, with all the

*Corresponding author at: Studio POZZI - International Center Oral Rehabilitation, Viale Liegi 44, Rome, Italy. interrelated anatomic parts involved in the patient smile (lips, cheeks, gingival architecture and teeth) is highly advised in order to deliver a successful facial and smile rejuvenation in the treatment of the aesthetic zone and even more important in the complete arch implant supported restorations [11-15].

The article introduces a more comprehensive, facially driven digital treatment plan to compose simulated patient models, through the superimposition of only 2 data sets of files the digital imaging and communications in medicine (DICOM) and the standard tessellation language (STL) files. The feasibility and advantages of this proof of concept digital integrated workflow are presented and discussed throughout the manuscript.

\section{Materials and methods}

An integrated digital workflow may enhance a more comprehensive treatment plan, based on a non-invasive simulation of the surgical and prosthetic outcomes, as well as a more effective communication among patient, clinician and dental technician $[16,17]$. The Smiling Scan technique allows the successful creation of a a virtual dental patient (VDP) showing a broad smile under static conditions, through the superimposition of 2 different digital 


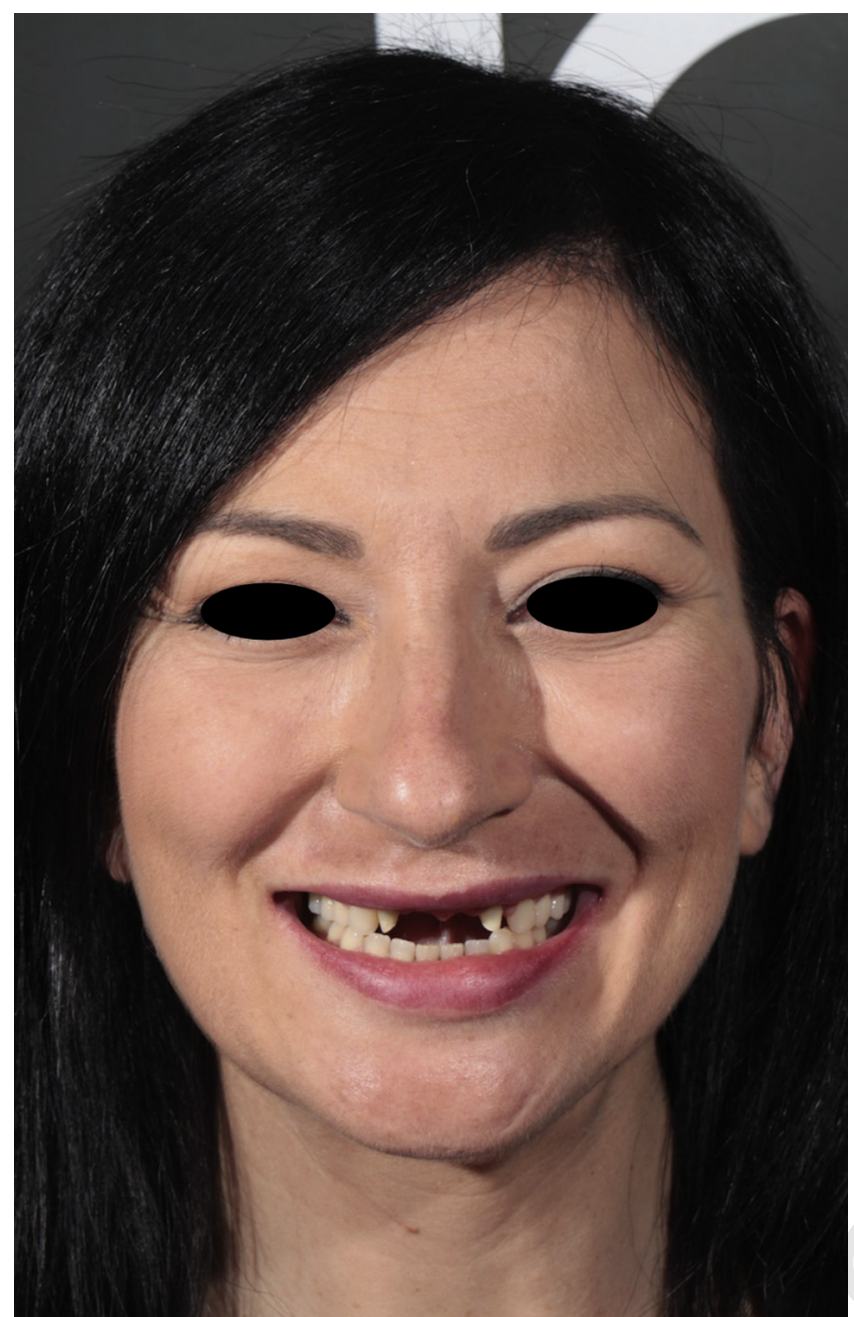

Fig. 1. The patient was displaying a broad smile during the CBCT examination.

data sets, the DICOM files generated by the cone beam computed tomography (CBCT) scan recorded while the patient displays a broad smile on for the duration of the scan and the STL files obtained by the intraoral optical surface scanning (IOS) or extraoral optical surface scanning (EOS) of the patient's intraoral anatomy (Carestream 3600 Intraoral Scanner, Carestream Dental LLC, Atlanta, GA, USA). The smile is a facial expression initiated by flexing the muscles surrounding the oral cavity (Obicularis oris) without vocalization thus displaying the front teeth. These instructions were given to the patient before taking the CBCT scan. A high speed CBCT device (Scanora 3Dx, Kavo Dental GmbH, Biberach, Germany) with an amorphous silicon detector was used to scan the patient with the following settings: field of view (FOV) $140 \mathrm{~mm}$ height, $100 \mathrm{~mm}$ width, high resolution (voxel sizes $0,25 \mathrm{~mm}$ ), $\mathrm{kV} \mathrm{90,} \mathrm{mA} \mathrm{10,} \mathrm{and} \mathrm{an} \mathrm{effective} \mathrm{exposure} \mathrm{time} 6 \mathrm{~s}$. The patient's head was properly secured on the CBCT scanner chair by means of the head frame positioner. The chin rest and support were not used to avoid any restrictions to the muscle movements during the smile or limiting the facial expression. The patient was asked to keep the dental arches in contact during the smile in order to record the current maxillo-mandibular skeletal relationship and occlusion (Figs. 1 and 2).

In case of patients with terminal dentition, the Smiling Scan is performed without any removable prostheses in the mouth. In the integrated digital workflow, the superimposition of the CBCT scan and optical surface scan, through matching of the resulting DICOM and STL data files, requires identifying corresponding landmarks in

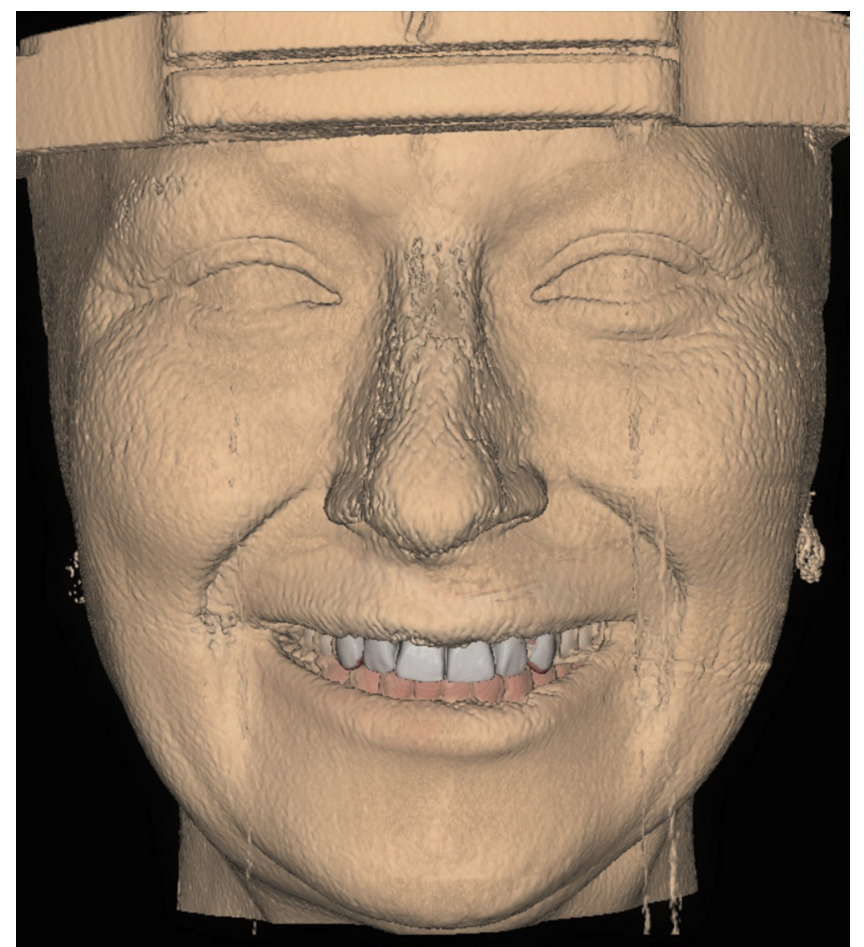

Fig. 2. The superimposition of the DICOM data and STL data was executed permitting the creation of a virtual dental patient.

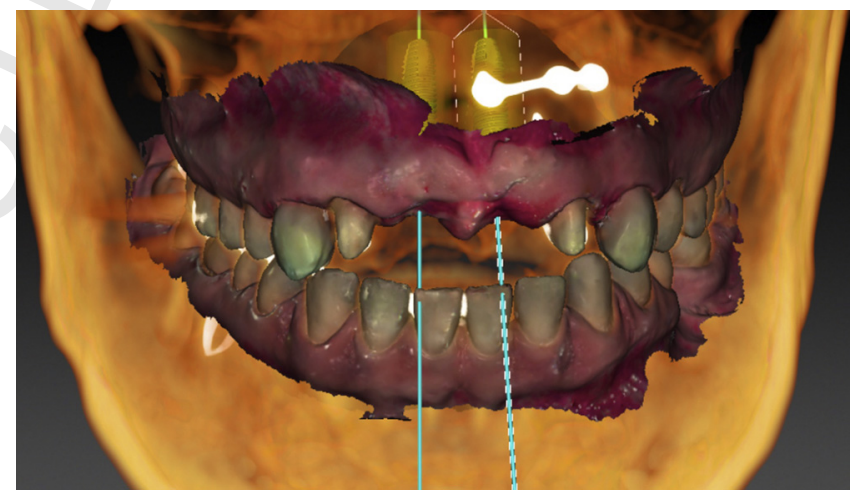

Fig. 3. The implant planning file is shared to the prosthetic software to design the temporary restoration.

both scanning datasets. The proprietary algorithm process (SmartFusion $^{\mathrm{TM}}$, Nobel Biocare AG) automatically overlays the DICOM data with the STL data, regardless if the source of the optical scanning is an intraoral scanner or a laboratory scanner. Technically, the accuracy of this automatic matching workflow is 1 voxel size below the manual segmentation workflow (Nobel Biocare AG, Kloten, Switzerland data on file), based on pairing of a minimum of three points on the surface of the patient's CBCT anatomy with the equivalent three points from the patient's anatomy obtained by digital high-resolution optical scanning. Thus, the combination of CBCT and IOS images, by superimposition of the data sets and use of planning software, provides a complete and accurate 3D representation of both hard and soft tissues (Fig. 3). The novel digital integrated workflow investigated by the authors is based on the automatic integration of two software, the surgical software (NobelClinician) and the restorative software (DTX Studio Design), (Nobel Biocare AG). The combination of the two software allowed the 3D implant planning according with the 


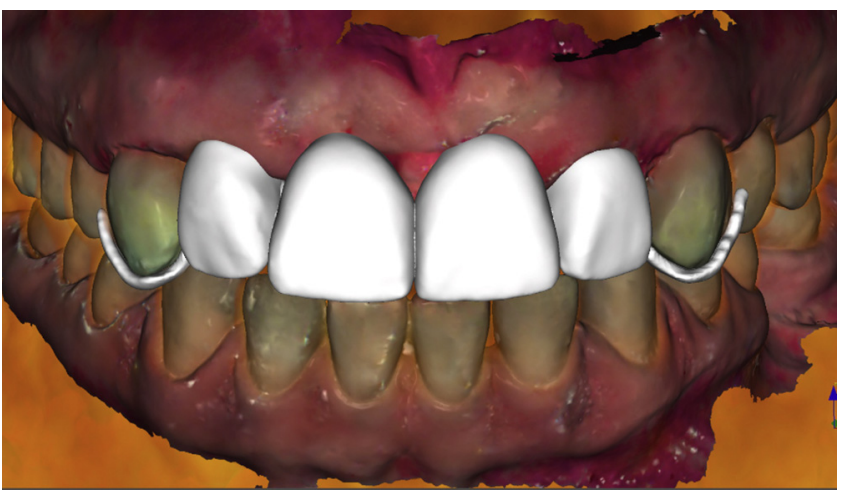

Fig. 4. The temporary restoration was designed with two lateral wings to facilitate the seating in the patient mouth according with the functional and aesthetic planning.

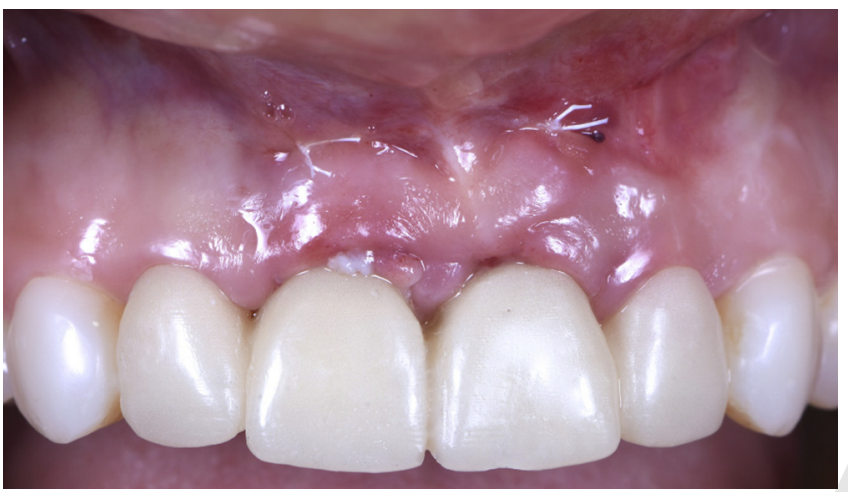

Fig. 5. CAD/CAM milled immediate provisionalization delivered the day of the surgery.

automated design of the missing tooth/teeth with the proper functional and esthetic shape, as well as placing the missing tooth/ teeth in occlusion with teeth in the opposing arch (Fig. 4). Moreover the prosthetic outcome can be verified and validated with the use of the fully-adjustable virtual articulator tool of the restorative software, properly set up according with the patient values assessed with an electronic face-bow (Arcus Digma, Kavo Dental GmbH).

In case of complete edentulism of one or both dental arches or terminal dentition with less than 3 teeth, the Smiling Scan was performed with a double scan technique [18].

\section{Difference from conventional methods}

Dentists face challenges in restoring aesthetics and function, particularly in case of patients with partial edentulism in the anterior zone, terminal dentition [19] or fully edentulism, due to the difficulties found with relating soft tissue facial features to the definitive casts used to identify the requirements of the intended restoration [11]. The smile design considers different scientific and artistic principles to create a pleasing smile for patients and often requires the evaluation of the patient's intraoral hard and soft tissue (teeth and gingival display) and their relationship to the facial features and smile [12,20-24]. Some authors proposed the use of extraoral 3D surface imaging systems and devices to incorporate a 3D virtual patient with realistic appearance into the preoperative diagnostic and treatment planning process. Harris et al. [25] suggested the use of a STL file from a portable 3D extraoral face scanner created at the patient's exaggerated smile to obtain the diagnostic information of the patient's maxillary lip

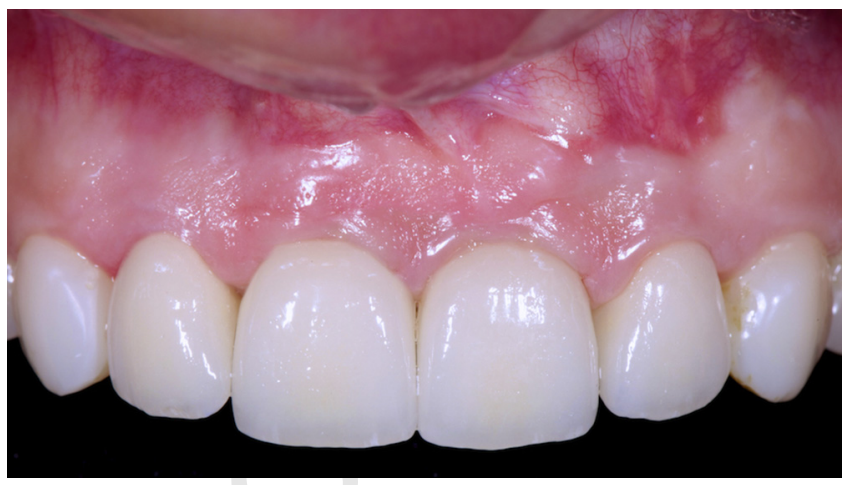

Fig. 6. Definitive restoration on site at 1 year follow up ( 4 porcelain fused to zirconia single fixed dental prostheses, screw retained onto the implants and cement retained onto the lateral incisors).

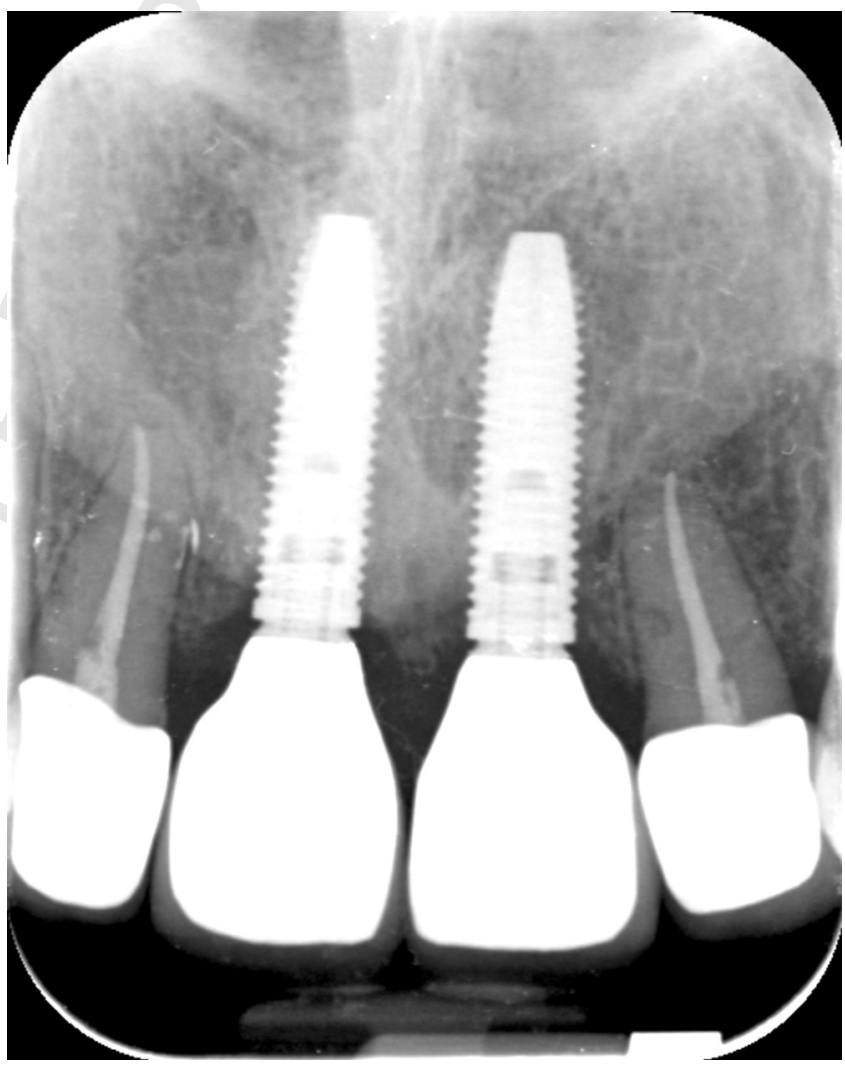

Fig. 7. Periapical radiograph of the definitive restorations at 1-year.

position and gingival display. However, differences in head positioning and facial expression during the data acquisitions can result in a registration error. The head positioning and facial expression must be firmly maintained during the acquisition process of 3D extraoral facial scanning and digital photography. Indeed, all the digital data sets to compose the 3D virtual patient should be merged or registered at a clinically acceptable level of accuracy. Furthermore, the current 3D optical facial scanners are cumbersome and expensive, and the file produced by such devices needs to be matched with the DICOM and STL file of the IOS/EOS scanning. This digital matching of 3 different data sets, to the best of our knowledge, still requires validation in terms of accuracy. The Smiling Scan technique allows the successful creation of a virtual dental patient showing a broad smile under static conditions, through the superimposition of only 2 different digital data sets, 


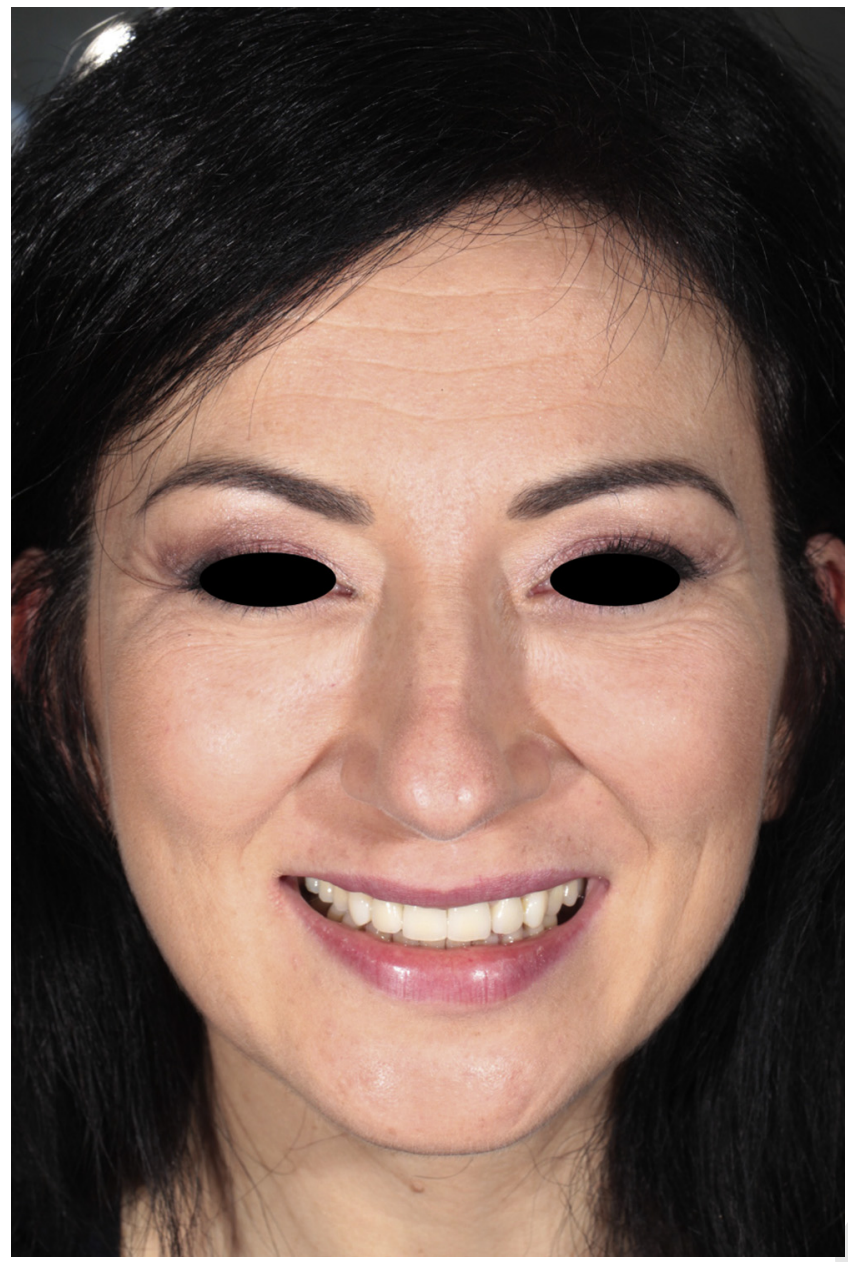

Fig. 8. The patient smile with the definitive restorations in situ reflects in shape and function the virtual tooth setup planned at the beginning of the treatment with the smiling scan technique. the DICOM and STL file, that represents the conventionally used digital integrated workflow. The patients did not experience any difficulties to keep the broad smile on for such short timeframe. However, the main limitation of this technique is represented by all the movement disorders and neurological tremors that cannot be controlled for the duration of the CBCT scan.

\section{Effect or performance}

The Smiling Scan technique allows the clinician to import all the information related to the patient's 3D facial anatomy while the patient is smiling using only one data set. Therefore, the patient's intraoral surface (gingival tissue and residual dentition) is scanned (STL files) and integrated with the craniofacial (hard tissue) model (DICOM files) to create a 3D visualization of the patient's hard and soft tissue anatomy. The smiling scan technique will allow the clinician to visualize the smile design of the patient and particularly the relationship between the upper, mid, and lower thirds of the face, the lines of symmetry, the lips, the cheeks and the residual dentition and to properly evaluate the aesthetic zone.

The smart set-up technology dramatically reduces the time it takes clinicians to create a prosthetic-driven treatment plan. Nevertheless, the effect on lip support from the virtual diagnostic tooth setup could not be simulated with the digital integrated workflow used by the authors due to software limitations. However, such limitations can be solved through the use of the double scan, digitally integrated workflow in which the Smiling

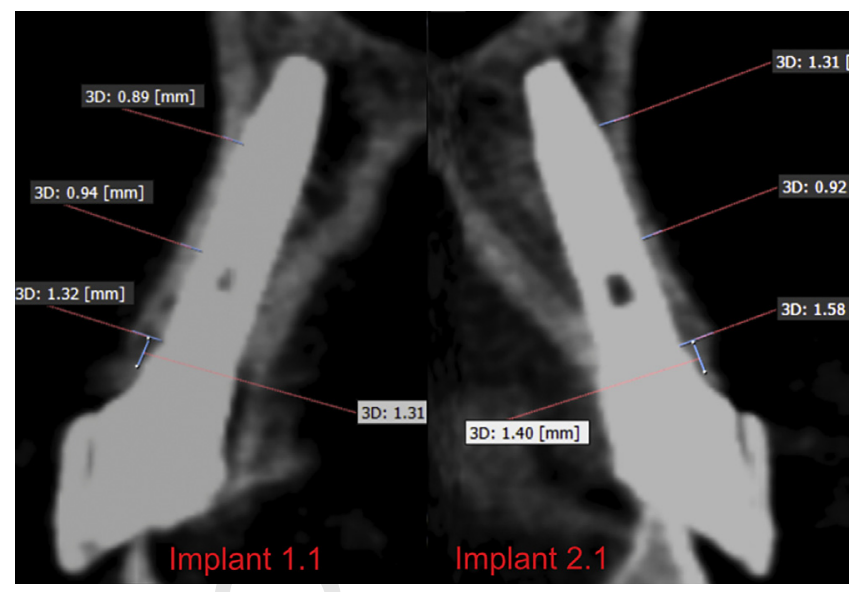

Fig. 9. СBCT cross section at 1 year follow up.

Scan technique is performed while the patient is wearing a radiographic guide properly addressing the maxillary lip support and smile set-up confirmed by the patient during the clinical tryin. The smiling scan technique does not look for a spontaneous smile but indeed it wants to record a broad smile as the maximum tooth and/or gingival display of the patient, in order to decide the implant positioning according with such relevant interplay between the lip position and the intraoral anatomy. Once the planning is completed and approved by the clinician, the digital information is used to produce the surgical template with computer aided manufacturing (CAM) rapid prototyping (3D printing), that will be tooth-supported in the case of a dentate patient or mucosa supported, in case of a fully edentulous patient. The Smiling Scan technique is not only a way to streamline the implant planning, but also generates a personalized computer aided design/computer aided manufacturing (CAD/CAM) interim restoration and definitive restoration (Figs. 4-9).

\section{Conclusions}

The smiling scan digital workflow is an effective, easy to use, and low-cost technique to enhance diagnostic capabilities and develop a more comprehensive facially and prosthetically driven implant treatment plan. The 3D virtual rendering of the cranio-facial and intraoral hard and soft tissues while the patient is smiling may enhance the decision making, addressing the functional and esthetic requirements of the patient and allowing a digitally assisted template guided implant placement and the delivery of an immediate CAD/CAM temporary fixed dental prostheses.

\section{References}

[1] Jacobs R, Adriansens A, Verstreken K, Suetens P, van Steenberghe D. Predictability of a three-dimensional planning system for oral implant surgery. Dentomaxillofac Radiol 1999;28:105-11.

[2] Klein M, Abrams M. Computer-guided surgery utilizing a computer-milled surgical template. Pract Proced Aesthet Dent 2001;13:165-9.

[3] van Steenberghe D, Naert I, Andersson M, Brajnovic I, Van Cleynenbreugel J, Suetens P. A custom template and definitive prosthesis allowing immediate implant loading in the maxilla: a clinical report. Int J Oral Maxillofac Implants 2002; 17:663-70.

[4] Vrielinck L, Politis C, Schepers S, Pauwels M, Naert I. Image-based planning and clinical validation of zygoma and pterygoid implant placement in patients with severe bone atrophy using customized drill guides. Preliminary results from a prospective clinical follow-up study. Int J Oral Maxillofac Surg 2003;32:7-14.

[5] Arunyanak SP, Harris BT, Grant GT, Morton D, Lin WS. Digital approach to planning computer-guided surgery and immediate provisionalization in a partially edentulous patient. J Prosthet Dent 2016;116:8-14.

[6] Pozzi A, Polizzi G, Moy PK. Guided surgery with tooth-supported templates for single missing teeth: a critical review. Eur J Oral Implantol 2016;9:135-53. 
G Model

JPOR $4651-5$

A. Pozzi et al./journal of prosthodontic research $x x x$ (2018) $x x x-x x x$

5

198

199

200

201

202

203

205

206

207
208

209

210

211

212

213

214

215

216

217
[7] Sennerby L, Anderson P, Pagliani L, Giani C, Moretti G, Molinari M, et al. Evaluation of a novel cone beam computed tomography scanner for bone density examinations in preoperative 3D reconstructions and correlation with primary implant stability. Chin Implant Dent Relat Res 2015;17:844-53.

[8] Rosenfeld AL, Mandelaris GA, Tardieu PB. Prosthetically directed implant placement using computer software to ensure precise placement and predictable prosthetic outcomes. Part 2: rapid-prototype medical modeling and stereolithographic drilling guides requiring bone exposure. Int J Periodontics Restorative Dent 2006;26:347-53.

[9] Rosenfeld AL, Mandelaris GA, Tardieu PB. Prosthetically directed implant placement using computer software to ensure precise placement and predictable prosthetic outcomes. Part 3: stereolithographic drilling guides that do not require bone exposure and the immediate delivery of teeth. Int J Periodontics Restorative Dent 2006;26:493-9.

[10] Pozzi A, Tallarico M, Marchetti M, Scarfò B, Esposito M. Computer-guided versus free-hand placement of immediately loaded dental implants: 1-year post-loading results of a multicentre randomised controlled trial. Eur J Oral Implantol 2014;7:229-42.

[11] Davis NC. Smile design. Dent Chin North Am 2007;51:299-318.

[12] Spear FM, Kokich VG. A multidisciplinary approach to esthetic dentistry. Dent Chin North Am 2007;51:487-505.

[13] Calamia JR, Levine JB, Lipp M, Cisneros G, Wolff MS. Smile design and treatment planning with the help of a comprehensive esthetic evaluation form. Dent Chin North Am 2011;55:187-209.

[14] Giannuzzi NJ, Motlagh SD. Full mouth rehabilitation determined by anterior tooth position. Dent Chin North Am 2015;59:609-21.

[15] Coachman C, Calamita MA, Coachman FG, Coachman RG, Sesma N. Facially generated and cephalometric guided 3D digital design for complete mouth implant rehabilitation: a clinical report. J Prosthet Dent 2017;117:577-86.
[16] Jodo T, Gallucci GO. The virtual patient in dental medicine. Chin Oral Implants Res 2015;26:725-6.

[17] Joda T, Bragger U, Gallucci G. Systematic literature review of digital threedimensional superimposition techniques to create virtual dental patients. Int J Oral Maxillofac Implants 2015;30:330-7.

[18] Verstreken K, Van Cleynenbreugel J, Martens K, Marchal G, van Steenberghe D, Suetens P. An image-guided planning system for endosseous oral implants. IEEE Trans Med Imaging 1998;17:842-52.

[19] Abdulmajeed A, Lim KG, Närhi TO, Cooper LF. Complete-arch implantsupported monolithic zirconia fixed dental prostheses: a systematic review. J Prosthet Dent 2016;115:672-7.

[20] Levin EI. Dental esthetics and the golden proportion. J Prosthet Dent 1978;40:244-52.

[21] Mack MR. Perspective of facial esthetics in dental treatment planning. J Prosthet Dent 1996;75:169-76.

[22] Bidra AS. Three-dimensional esthetic analysis in treatment planning for implant-supported fixed prosthesis in the edentulous maxilla: review of the esthetics literature. J Esther Rester Dent 2011;23:219-36.

[23] Vasantha Kumar M, Ahila SC, Suganya Devi S. The science of anterior teeth selection for a completely edentulous patient: a literature review. J Indian Prosthodont Soc 2011;11:7-13.

[24] Free C, Staehle HJ, Wolff D. The assessment of dentofacial esthetics in restorative dentistry: a review of the literature. J Am Dent Assoc 2012;143:461-6.

[25] Harris BT, Montero D, Grant GT, Morton D, Lop DR, Lin WS. Creation of a 3dimensional virtual dental patient for computer-guided surgery and CAD-CAM interim complete removable and fixed dental prostheses: a clinical report. J Prosthet Dent 2017;117:197-204.
218

219

220

221

222

223

224

225

226

227

228

229

230

231

232
233

Please cite this article in press as: A. Pozzi, et al., The smiling scan technique: Facially driven guided surgery and prosthetics, J Prosthodont Res

(2018), https://doi.org/10.1016/j.jpor.2018.03.004 